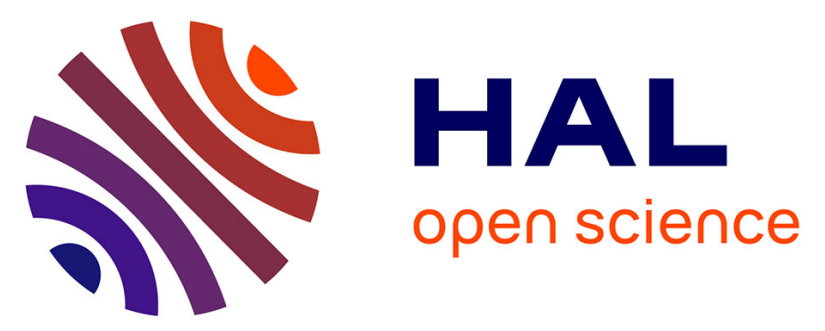

\title{
Efficient Experimental Assessment of The Specific Absorption Rate (SAR) Induced by MIMO Wireless Communication Devices; Application of Vector Near-Field Measurement System
}

Lyazid Aberbour, Ourouk Jawad, Mehdi Ramdani, Philipe Giry, Thomas Julien

\section{To cite this version:}

Lyazid Aberbour, Ourouk Jawad, Mehdi Ramdani, Philipe Giry, Thomas Julien. Efficient Experimental Assessment of The Specific Absorption Rate (SAR) Induced by MIMO Wireless Communication Devices; Application of Vector Near-Field Measurement System. 2018 IEEE Conference on Antenna Measurements \& Applications (CAMA), Sep 2018, Västerås, Sweden. pp.1-4, 10.1109/CAMA.2018.8530621. hal-02376333

\section{HAL Id: hal-02376333 \\ https://hal.science/hal-02376333}

Submitted on 22 Nov 2019

HAL is a multi-disciplinary open access archive for the deposit and dissemination of scientific research documents, whether they are published or not. The documents may come from teaching and research institutions in France or abroad, or from public or private research centers.
L'archive ouverte pluridisciplinaire HAL, est destinée au dépôt et à la diffusion de documents scientifiques de niveau recherche, publiés ou non, émanant des établissements d'enseignement et de recherche français ou étrangers, des laboratoires publics ou privés. 


\section{Efficient Experimental Assessment of The Specific Absorption Rate (SAR) Induced by MIMO Wireless Communication Devices; Application of Vector Near- Field Measurement System}

\author{
Lyazid Aberbour \\ ART-Fi \\ Orsay, France \\ lvazid.aberbour@art
}

\author{
Ourouk Jawad \\ ART-Fi \\ Orsay, France \\ ourouk.jawad@art-
}

\author{
Mehdi Ramdani \\ ART-Fi \\ Orsay, France \\ mehdi.ramdani@art- \\ fieu
}

\author{
Philipe Giry \\ ART-Fi \\ Orsay, France \\ philipe.giry@art- \\ fieu
}

\author{
Thomas Julien \\ ART-Fi \\ Orsay, France \\ thomas.julien@art-
}

\begin{abstract}
This paper introduces an efficient procedure for the evaluation of the true exposure level, quantified by the peak averaged specific absorption rate (SAR), of multiple-input multiple-output (MIMO) communication devices operating transmission diversity. As compared to the traditional conservative evaluation of the SAR, that results from the unpractical testing of all the antenna-array states of the device, the assessment of the true exposure level enables higher margin for minimizing the SAR while maximizing the radiation performance of the device.
\end{abstract}

Keywords-SAR, MIMO, Planar Near-Field Measurement System, Vector Field Measurement.

\section{INTRODUCTION}

With the recent evolutions of wireless technology standards, multiple-input multiple-output (MIMO) technology is becoming popular in wireless communication devices and user equipment. Such devices that are intended for usage in proximity to the user are subject to regulations on their level of human exposure to their electromagnetic field radiation. For frequencies below $6 \mathrm{GHz}$, the specific absorption rate (SAR) is a widely recognized measurand of the human exposure, and its assessment is standardized [1-3], especially for its assessment from the internal electric field power measurement in human tissue equivalent media.

The particularity of MIMO antenna systems in exposure compliance testing is their unpractical high-number of the array states that need to be tested for the evaluation of the true SAR level. N. Perentos, et al [4], proposed several summation schemes of the amplitude only of the internal electric field that provide conservative estimates of the true SAR level of MIMO devices, all with reasonable measurement duration. One shortcoming of conservative exposure evaluation approaches is the resulting underestimation of the maximum permitted radiation power, which in turn might result in an underexploitation of the MIMO capability.
D. T. Le, et al [5], introduced a fast procedure for estimating the true SAR of MIMO devices through direct measurements, or phase retrieval techniques, of the electric-field phasor vector. The introduced method for SAR evaluation of an $N$-antenna device, requires $N(N-1)+1$ measurements if only the amplitude of the electric field measurement is possible, but uses only $N$ measurements if the electric-field phasor vector is directly measured. The later, measurement typically requires a phase reference wave which is independent from the electromagnetic field of the device under test (DUT).

In this paper, a fast and efficient procedure is introduced for the assessment of the MIMO-SAR on a vector SAR measurement system, but that takes as a phase reference-wave the electric field of the antenna under test for measuring the electric field phasor vector. It uses the measurements of the vector electric-field data from the individual antenna-array elements, and at least an additional measurement of simultaneous excitation of the antenna-array. The true SAR level of the MIMO system is then assessed through postprocessing by linearly combining the individual field data weighted by all the array configuration coefficients.

This paper is organized as follows. Section II introduces the vector field measurement system, as well as the experimental set-up. The vector measurement-based MIMO-SAR procedure is detailed in section III, and the experimental validation results are reported in section IV, followed by conclusions in section V.

\section{EXPERIMENTAL SET-UP}

\section{A. Overview of the measurement system}

ART-MAN is a planar near-field measurement system which enables measurement of the time-independent complex E-field phasor vector in a broadband human tissue simulant material. The time-domain vector E-field probe array consist in a grid of dual-polarized sensors made for capturing two orthogonal E-field components tangential to the measurement surface. Near-field vector techniques directly exploit the 
harmonic property of the electromagnetic field. A corollary of this property is that the knowledge of amplitude and phase of E-field components ( $E_{x}$ and $E_{y}$ ) tangential to a surface is enough to deduce the three E-field components $(x, y, z)$ in all three dimensions [6-7].

Hence, measuring vector fields makes it possible to define a simple arrangement for an array of probes, which enables an accurate electric field evaluation in the volume of the mannequin, with no need for approximating the propagation. Assessing vector fields requires determining the phase relative to a reference. Relative phase of the electric field vector on the probe array grid is referenced to the phase of the electric field of a preselected probe element among the probe array [6]. Referencing of the phase of the electric vector is achieved through synchronous and coherent acquisition of the probe voltage time-series on the measurement and the reference probes through a two-way RF chain.

\section{B. Experimental set-up}

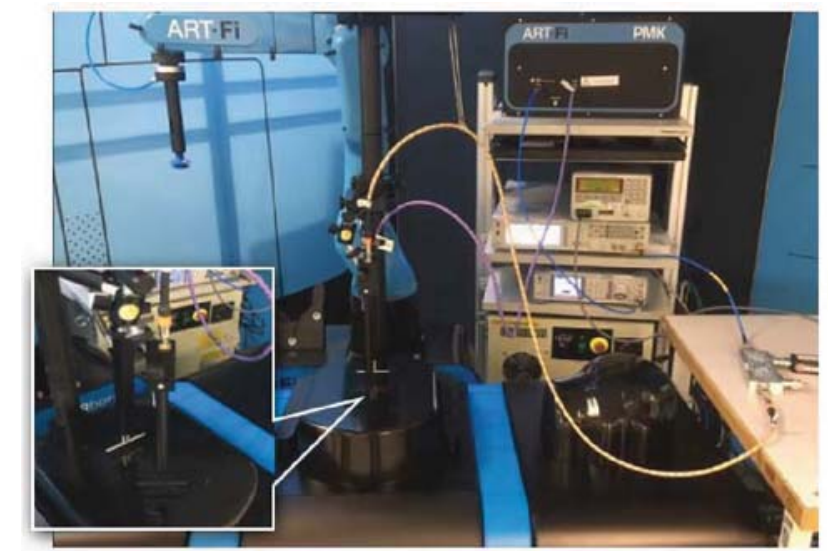

Fig. 1 Photography of the experimental set-up; two dipole antennas placed on top of the flat phantom of ART-MAN (vector SAR measurement system).

The experimental MIMO demonstrator set-up is composed by two dipole antennas operated at $1.9 \mathrm{GHz}$. The distance between the centers of the two dipoles is $0.5 \lambda$. Dipole- 1 orientation is $0^{\circ}$ and Dipole- 2 orientation is $45^{\circ}$. The distance between the dipoles and ART-MAN's flat phantom is $15 \mathrm{~mm}$. Both dipoles are connected to two different signal generator and the forward power at dipoles port is monitored thanks to two directional couplers. The forward power is $17 \mathrm{dBm}$ for both dipoles. Rohde \& Schwarz SMB 100A signal generator is connected to dipole 1 and Agilent EXG N5172B is connected to dipole 2. The local oscillator synchronization enables control of the phase shift between the two continuous wave (CW) signals.

\section{DESCRIPTION OF THE SAR ASSESSEMENT PROCEDURE|}

The volume SAR distribution assessment in the herein proposed method is performed using the induced time-harmonic electric-field power expression, which reads at a measurement point $P(x, y, z)$ :

Identify applicable funding agency here. If none, delete this text box

$$
\operatorname{SAR}(x, y, z)=\frac{\sigma|\boldsymbol{E}(x, y, z)|^{2}}{\rho}\left\lceil\frac{\mathrm{W}}{\mathrm{kg}}\right\rceil
$$

where $\sigma$ is the electric conductivity $[\mathrm{S} / \mathrm{m}]$ and $\rho$ is the mass density $\left[\mathrm{kg} / \mathrm{m}^{3}\right]$ of the human tissue simulant media. The phasor of the induced electric-field vector $\boldsymbol{E}(x, y, z)$ of the multiple-antenna device is the vector summation of the individual phasors of the electric fields from each antenna element $\left(\boldsymbol{E}_{i=1: N}\right)$ of the N-antenna array of elementary feed weights $w_{i}$, it can be expressed as

$$
\boldsymbol{E}(x, y, z)=\sum_{i=1}^{N} w_{i} \cdot \boldsymbol{E}_{i}(x, y, z)\left[\frac{\mathrm{V}}{\mathrm{m}}\right]
$$

LO synchronisation

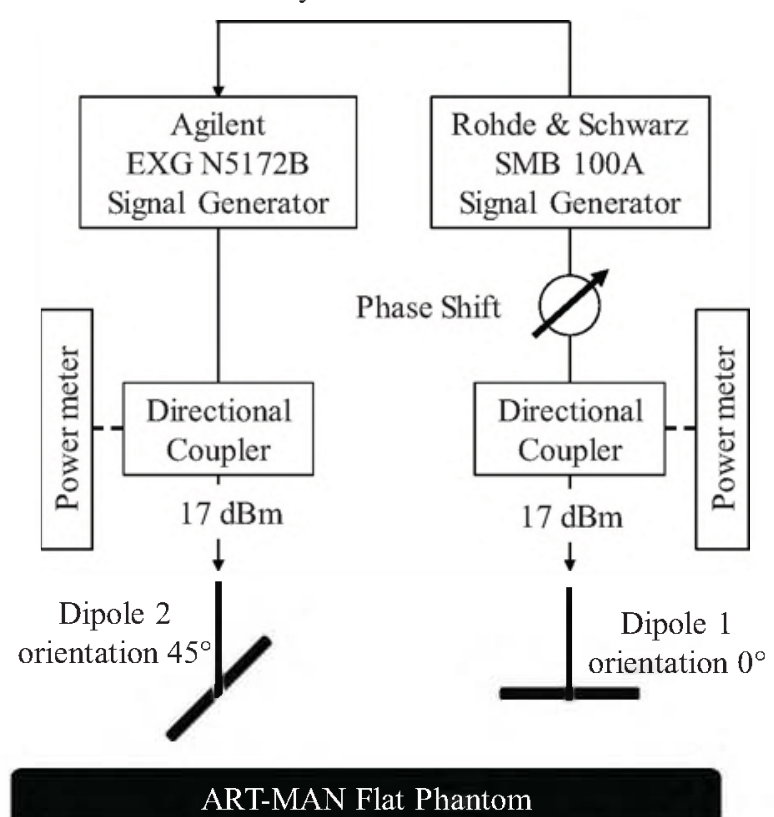

Fig. 2 Block diagram of the experimental set-up

It is apparent from (2) that for SAR compliance testing though assessment of the actual maximum realizable volumeaveraged SAR, an equal number of measurements as the array interference states is needed, especially when considering scalar based measurement systems, which take measurements of the RMS value of the electric field vector. On vector-based measurement systems, which take measurements of the complex time-independent field vector components, one might take measurements, or reconstruct out of a finite number of spatial samples, the individual field data $\boldsymbol{E}_{i}(x, y, z)$ for arbitrary known weighting coefficients $w_{i}$, then superpose the data to retrieve the total field distribution for all the antenna-array interference states, that might be tabulated in a code-book.

As explained in section II.A, for the specific application of active-device testing on ART-MAN measurement system, the individual field data $\boldsymbol{E}_{i}(x, y, z)$ from each antenna element of the DUT are with independent wave references. Actually, when taking a field scan on the system probe-array, the acquired timedomain voltages on the probe array are all with a wave reference 
that is provided by a probe which is selected among the probearray and is hence kept at a fixed-distance from the DUT during the complete scan of the probe-array. For a given DUT at a fixed position from the probe-array, a change in the location of the phase-reference probe only introduces a constant phase shift on the electric field samples of the probe array.

A mean for referencing the relative phase of the field data $\boldsymbol{E}_{i}(x, y, z)$ of the individual antenna elements of the DUT consists in single or multiple additional measurements of the total field $\boldsymbol{E}(x, y, z)$ from simultaneous excitation of the antenna-array of the DUT, but with known phase-shifts and magnitude of the feed coefficients. The magnitude of the so obtained total electric field $|\boldsymbol{E}|$ can then be employed to estimate the phase-shifts to be applied on the individual phasor data $\boldsymbol{E}_{i}$.

Considering for instance the experimental set-up as illustrated in Fig. 2, the main steps of the proposed procedure for the assessment of the actual peak volume-averaged SAR for the two-antenna MIMO system are with five main steps.

- Step\#1: The vector E-field for sequential excitation of each one of the two antenna ports are measured one after the other, while the other port is terminated with matched load, for an equal and monitored incident power. We hence obtain at the probe plane, defined by $z=z_{0}$, $\boldsymbol{E}_{1}\left(x, y, z_{0}\right)$ for the excitation at Dipole-1's port and $\boldsymbol{E}_{2}\left(x, y, z_{0}\right)$ for the excitation at Dipole-2's port.

- Step\#2: The vector E-field for simultaneous excitation at all of the two antenna ports is measured for equal incident power and an enforced phase shift $\varphi_{0}$ on the two signal's generators. We obtain at the probe plane a combined total field $\boldsymbol{E}_{\mathrm{cmb}}\left(x, y, z_{0}\right)$.

- Step\#3: The field measurement results of Step\#1 and Step $\# 2$ are employed to retrieve the phase-shift $\beta$ that minimizes the RMSE on the amplitude of the measured $\left|\boldsymbol{E}_{\mathrm{cmb}}\right|$ and superposed $\left|\boldsymbol{E}_{1}+\boldsymbol{E}_{2, p} \cdot e^{j\left(\varphi_{0+\beta}\right)}\right|$ of reference the relative phase of the two E-field measurements in Step\#1, according to the following expression

$$
\begin{aligned}
& \min _{\beta} \| \sqrt{\left|\sum_{p=x, y, z}\right| E_{1, p}\left(x, y, z_{0}\right)+\left.E_{2, p}\left(x, y, z_{0}\right) \cdot e^{j\left(\varphi_{2}+\beta\right)}\right|^{2}} \\
& -\sqrt{\sum_{p=x, y, z}\left|E_{\mathrm{cmb}, p}\left(x, y, z_{0}\right)\right|^{2}} \|
\end{aligned}
$$

- Step\#4: The total E-field from the antenna array at it's all interference states, defined by the complex weights $\left(\alpha_{i=1: M}, \gamma_{i=1: M}\right)$ of the antenna array ports, are computed as

$$
\begin{gathered}
\boldsymbol{E}_{\mathrm{cmb}, i}(x, y, z)=\sum_{i=1}^{M} \alpha_{i} \cdot \boldsymbol{E}_{1}(x, y, z) \\
+\gamma_{i} . \boldsymbol{E}_{2}(x, y, z) e^{j \beta}
\end{gathered}
$$

- Step\#5: For each array interference state $i=1 ; M$, the volume SAR is computed and the 1-gram cube and 10-gram cube averaging algorithm [3] is employed to evaluate the actual peak volumeaveraged SAR of the MIMO system.

\section{EXPERIMENTAL VALIDATION RESULTS}

The field measurement data for the individual port excitations are shown in Fig., and in for the measurement of
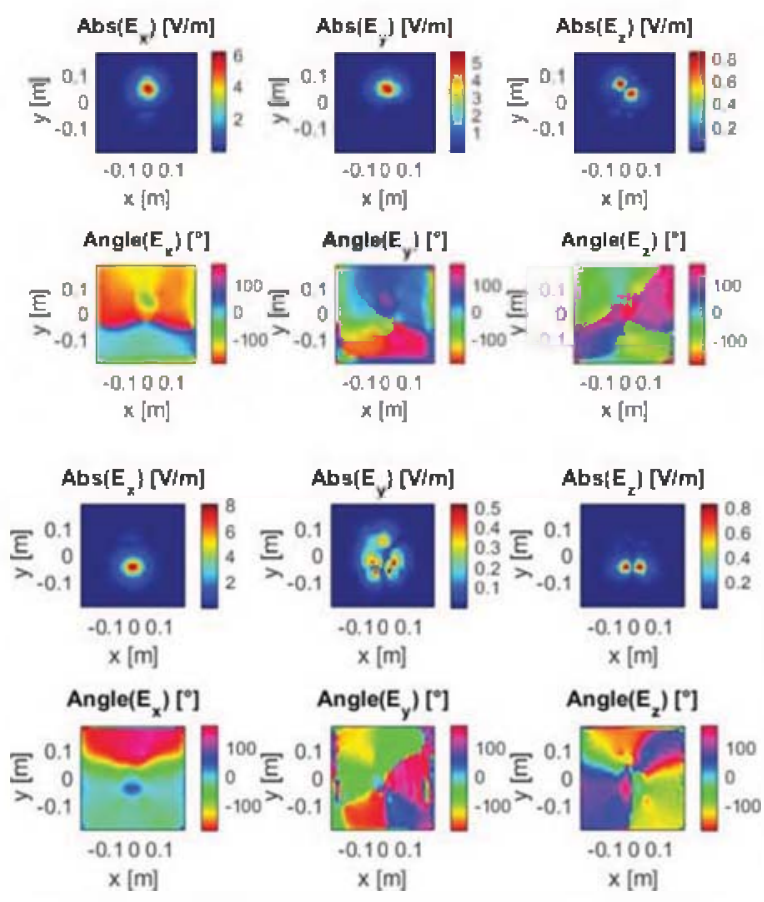

Fig. 3 Plots of the amplitude and relative phase of the electric-field for excitation at Dipole-1 port (Top) and at Dipole-2 port (Bottom)

the simultaneously fed dipoles, with a phase shift of $3 \pi / 4$. The retrieved E-field results using Eq. (4), according to the methodology of section III, is reported in Fig. .

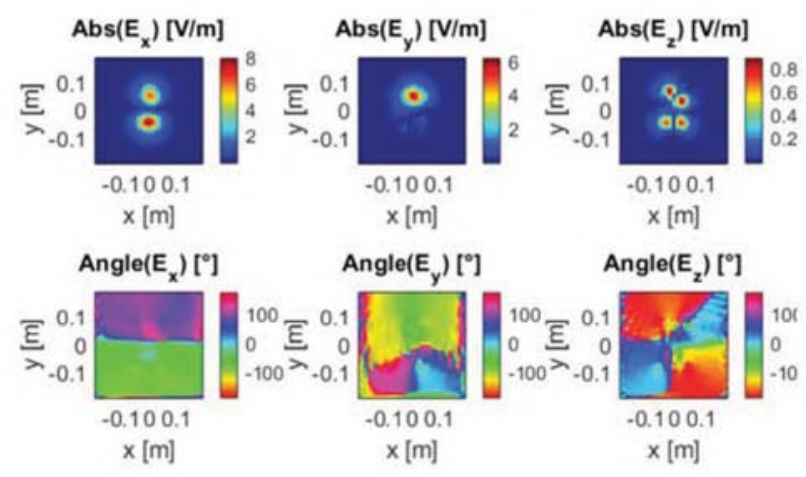

Fig. 4 Plots of the E-field measurement data for simultaneous feed of the dipoles with $3 \pi / 4$ phase shift. 
It is visible from the results in Fig. that the proposed approach is very accurate and highly efficient. An error of less than $4 \%$

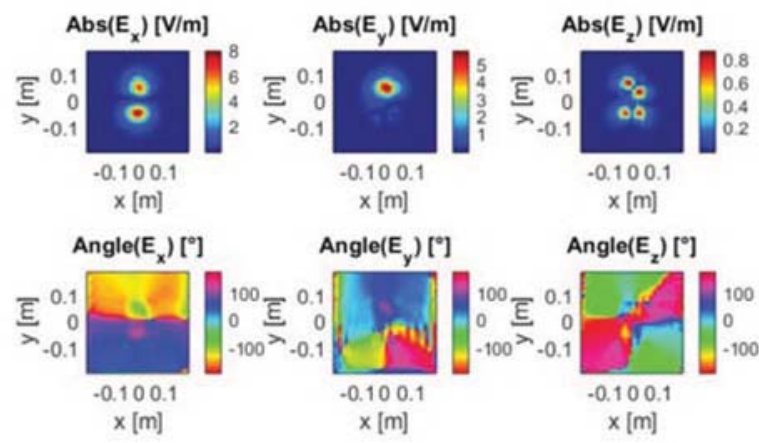

Fig. 5 Results for the retrieved E-field using Eq. (4), and the measurements of the individual antennas.
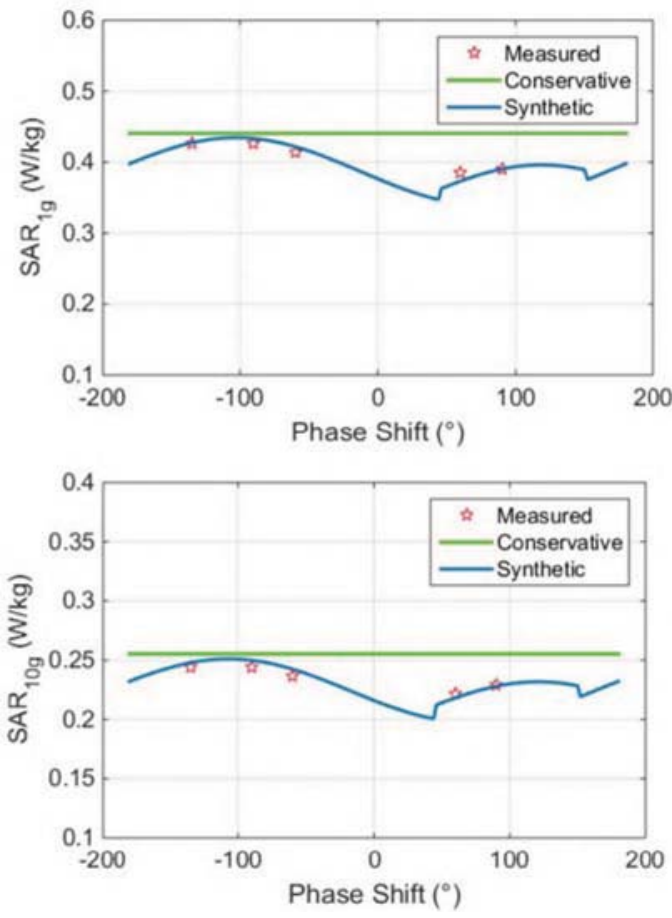

Fig. 6 Results of peak 1-gram and 10-gram averaged SAR obtained from, conservative evaluation of SAR (green), direct measurement of the simultaneously fed antennas ( $\mathrm{red}$ ), and from

the retrieved total E-field from the individual antenna measurements (bleu) using Eq. (4).

is obtained on the SAR values from the retrieved total field data w.r.t the direct total field measurement for the tested phaseshifts on the two antenna feed weights.

\section{CONCLUSIONS}

This paper presented an efficient procedure for the evaluation of the true exposure level, quantified by the SAR level, of MIMO devices operating beamforming, hence enabling higher margin for minimizing the device SAR level while maximizing its radiation and performance. The efficiency of the procedure is due to the measurement of the timeindependent vector phasor of the electric field radiated by the device under test. It especially addresses the case of near field measurement systems that take a phase-reference wave which is not independent from the electromagnetic field radiated by the antenna under test. It should be sufficient to take $\mathrm{N}+1$ number of measurements for the assessment of the MIMO SAR of a $\mathrm{N}$ antenna device.

Further work includes development of test procedures for MIMO devices that are with high number of antenna elements, of exclusively simultaneous-excitation schemes with arbitrary amplitude and phase weightings of the array, as well as the development of SAR test methods for the emerging fast switching MIMO technologies.

\section{ACKNOWLEDGMENT}

The authors are acknowledgeable to the EURAMET for funding the Vector SAR project 16NRM07.

\section{REFERENCES}

[1] ICNIRP, "Guidelines for limiting exposure to time-varying electric, magnetic and electromagnetic fields (up to $300 \mathrm{GHz}$ )," Health Phys., vol 74, pp. 494-522, 1998

[2] IEC 62209-2, "Human exposure to radio frequency fields from hand-held and body-mounted wireless communication devices - Human models, instrumentation, and procedures Part 2: Procedure to determine the specific absorption rate (SAR) for wireless communication devices used in close proximity to the human body (frequency range of $30 \mathrm{MHz}$ to 6 GHz)", Ed. 1.0, 2010.

[3] IEEE 1528, "IEEE Recommended Practice for Determining the Peak Spatial-Average Specific Absorption Rate (SAR) in the Human Head from Wireless Communications Devices: Measurement Techniques", Ed. 2013.

[4] N. Perentos, S. Iskra, A. Faraone, R. J. McKenzie, G. Bit Babik and V. Anderson, "Exposure Compliance Methodologies for Multiple Input Multiple Output (MMO) Enabled Networks and Terminals," in IEEE Transactions on Antennas and Propagation, vol. 60, no. 2, pp. 644-653, Feb. 2012

[5] D. T. Le, L. Hamada, S. Watanabe and T. Onishi, "A Fast Estimation Technique for Evaluating the Specific Absorption Rate of MultipleAntenna Transmitting Devices," in IEEE Transactions on Antennas and Propagation, vol. 65, no. 4, pp. 1947-1957, April 2017.

[6] B. Derat et al., "A novel technology for fast and accurate specific absorption rate measurement," 2013 International Workshop on Antenna Technology (iWAT), Karlsruhe, 2013, pp. 363-366.

[7] B. Derat, L. Aberbour and A. Cozza, "Near-field and vector signal analysis techniques applied to specific absorption rate measurement," 2015 IEEE MTT-S 2015 International Microwave Workshop Series on RF and Wireless Technologies for Biomedical and Healthcare Applications (IMWS-BIO), Taipei, 2015, pp. 34-35

[8] Patent WO/2011/080332, System For Measuring An Electromagnetic Field. 